\title{
Image Color Cube Dimensional Filtering and Visualization
}

\author{
Jose-Luis Lisani ${ }^{1}$, Antoni Buades ${ }^{2}$, Jean-Michel Morel ${ }^{3}$ \\ 1 TAMI, Universitat Illes Balears, Spain (joseluis.lisani@uib.es) \\ ${ }^{2}$ MAP5, Université Paris Descartes, France (toni.buades@uib.es) \\ ${ }^{3}$ CMLA, ENS Cachan, France (moreljeanmichel@gmail.com) \\ Communicated by Julie Delon Demo edited by Jose-Luis Lisani
}

\begin{abstract}
We call RGB cube the three dimensional representation of the colors of a digital image in RGB space. It has been observed that $2 \mathrm{D}$ structures predominate in the color cube. They are qualitatively explained by two main causes: image blur and texture. In this article a filtering algorithm, the Local Linear Projection (LLP2), is used to smooth the 3D color distribution and to reveal the underlying $2 \mathrm{D}$ structures of the color clusters.
\end{abstract}

\section{Source Code}

The source code (ANSI C), its documentation, and the online demo are accessible at the IPOL web page of this article ${ }^{1}$.

Keywords: color cube; filtering; 2D structures

\section{Overview}

We call RGB cube the three dimensional representation of the colors of a digital image in RGB space. This cube simply shows the $(R, G, B)$ color points which actually appear in the digital image. This visualization has, however, two main defects: it does not show the real RGB histogram. The difference is that in the color cube each point is plotted with its own color, while in the RGB histogram it has a grey level representing the number of pixels in the image having this color value. It is of course impossible to display these two features simultaneously. The perspective views, which are most used, hide the real dense cloud, which is surrounded by a fume of isolated color points. These isolated color points, mainly due to the image noise and to compression artifacts, make an opaque obstacle to the perspective view of the cloud. Perspective views remain nonetheless the classic visualization tool, because the cube occupation by the color points of a natural image is far from complete, as it can be seen in the example in Figure 1.

\footnotetext{
${ }^{1}$ https://doi.org/10.5201/ipol.2011.blm-cdf
} 

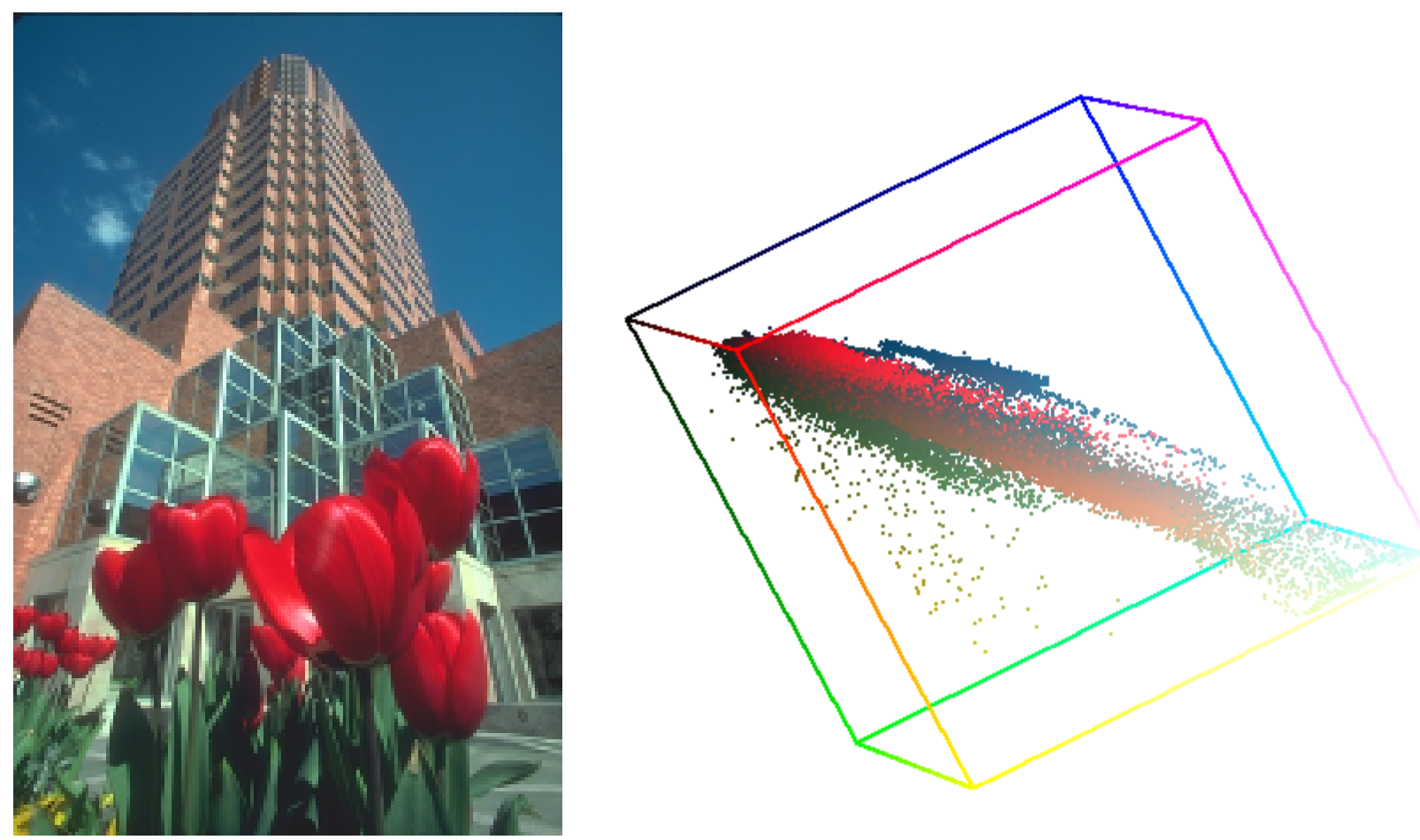

Figure 1: Original image and its color cube.

In [1] the authors give a comprehensive understanding of all spatial structures appearing in a color histogram. They explore the building parts of the color cube by a qualitative analysis of image details. They also investigate how these elementary structures are combined, and their physical causes. Their conclusion is that 2D structures predominate in the color cube. They are qualitatively explained by two main causes: image blur and texture.

Besides the qualitative analysis of spatial structures in RGB space, the authors of [1] make a quantitative evaluation supporting the $2 \mathrm{D}$ dimensional character of the RGB cube. To this aim a filtering algorithm, the Local Linear Projection (LLP2) is used to smooth the color distribution and to reveal the underlying $2 \mathrm{D}$ structures of the color clusters.

LLP2 replaces each RGB color in the cube by its projection onto a locally defined regression plane, computed by Principal Components Analysis (PCA). The process is iterated some two to four times, until the average error between corresponding colors in consecutive iterations is below a threshold of 0.5 .

Thus LLP2 estimates a 2D manifold from the unstructured cloud of points allowing for a better visualization of the colors. This $2 \mathrm{D}$ manifold is usually very close to the initial cloud: on average the displacement of the color points is around 3 (see Table 3), which is quite small in relation to the cube dimensions $(255,255,255)$. On this manifold, the color density is displayed by a brightness value proportional to its logarithm. The density has become visible because the cloud is flattened and the dense color points in direct sight, being no more hidden by low density color points (see Figure 2).

The algorithm is described in the next sections, together with implementation details. Moreover, several examples are displayed. 


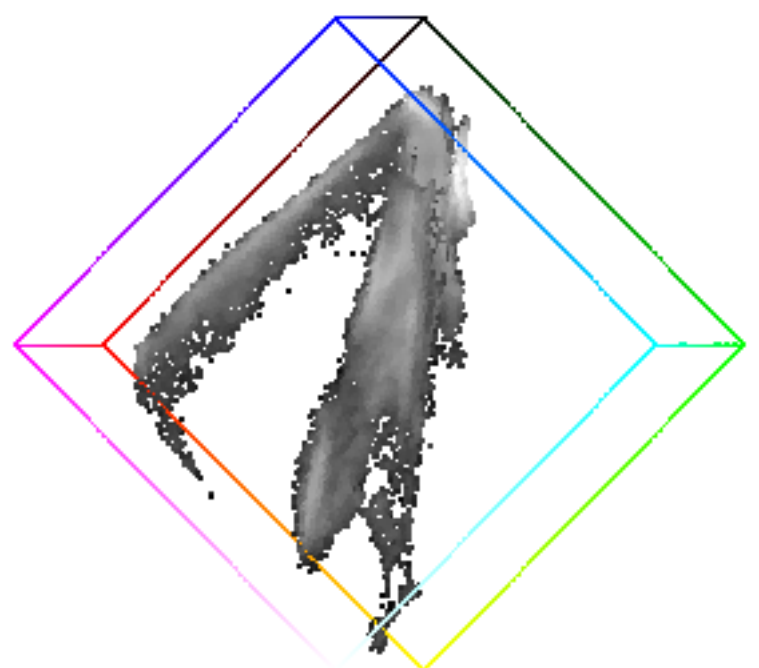

Figure 2: Perspective view of the color cube of the image in Figure 1 after filtering with LLP2. Each color is displayed with a grey level proportional to the logarithm of the density in RGB space (lighter for higher densities). The density of color points is defined in Section 7.1.

\section{Algorithm}

Given a $k$-dimensional manifold given by noisy samples, the problem of recovering the manifold by denoising the samples and meshing them is a classic problem whose solution can be taken advantage of for processing color clouds. The founding method seems to be the moving least square (MLS) method introduced in [2]. It is proved in [3] that the MLS method of order 1, which projects the point cloud locally on its regression plane, moves the underlying surface by mean curvature motion. This means that if the surface is flat, it hardly moves by MLS1. However, if the point cloud is noisy, a smooth manifold is fast restored and can be meshed, as proved in [3]. MLS1 is a particular case of the Local Linear Projection (LLP) algorithm [4], which does not assume a particular dimension. LLP with $k$ dimensions (LLP $k$ ) takes for each point its closest points in Euclidean distance and computes the PCA of this neighborhood. Then, the current point is projected on the regression affine manifold generated by the $k$ first PCA components. This algorithm was proposed by Huo et al. [4] for the filtering of high dimensional data. Notice that MLS1 and LLP2 are the same algorithm.

The adaptation of LLP to the color point clouds is described below. Each RGB color actually may represent more than one pixel in the image. When applying this filtering strategy each color is counted as many times as it appears in the image.

\section{LLP algorithm.}

For each RGB color $y_{i}, i=1,2, \cdots, N$,

1. Find the $K$ neighbors whose distance to $y_{i}$ is smaller than a given threshold $T$. The neighboring colors are denoted by $x_{1}, x_{2}, \cdots, x_{K}$.

2. Compute the principal components of the set $x_{1}, x_{2}, \cdots, x_{K}$.

3. Let $k(k=0, k=1$ or $k=2)$ be the assumed dimension of the embedded manifold, then project $y_{i}$ onto the affine subspace spanned by the first $k$ principal components and passing through the center of mass of the set $x_{1}, x_{2}, \cdots, x_{K}$. 
The algorithm is iterated until the average difference between corresponding colors in two consecutive iterations is below a precision parameter $\varepsilon$.

In practice only LLP2 is used, since the $2 \mathrm{D}$ model has proven [1] to be the best adapted to the color distributions found in natural images.

\section{Implementation Details}

The complexity of the algorithm is a function of the number of colors $C$ in the image. For each color, a neighborhood of size $(2 T+1)^{3}$ is explored (step 1 of the algorithm) and then the color point is processed. This simple analysis leads to an upper bound of $O\left(C T^{3}\right)$ operations per iteration. The total number of iterations depends on the speed of convergence of the algorithm.

It is not possible to give an a priori estimate of the computation time in terms of the size of the image, since there is no exact correspondence between number of pixels and number of colors. Statistical analysis performed on 100 images from the Berkeley test dataset [5] (size $481 \times 321)$ gives an average ratio of 4.24 pixels/color. Moreover, this ratio decreases as the image size is reduced (see Table 1).

$\begin{array}{cccc}\text { Scale } & \text { Size (pixels) } & \text { Colors } & \text { Pixels/Color } \\ 1 & 154401 & 36363.13 & 4.24 \\ 0.75 & 86400 & 26676.66 & 3.24 \\ 0.5 & 38400 & 16482.54 & 2.33 \\ 0.25 & 9600 & 6155.44 & 1.56\end{array}$

Table 1: Number of pixels and average number of colors computed from 100 images from the Berkeley test dataset [5] for different scale factors. The last column shows the ratio between the number of pixels with respect to the number of colors.

The following specific implementation of the LLP algorithm has been adopted in order to reduce the computation time while ensuring the quality of the results:

- in step 1, L1 distance is used and the value of threshold $T$ is fixed to 10. In the Examples section it is shown that the value of this parameter is not critical for the algorithm results, provided that the right model of color distribution (2D model) is used.

- in step 2, the minimum number $K$ of neighbors required for principal components analysis is set to 10, therefore the algorithm is not applied to isolated points in RGB space. These points keep their initial RGB value throughout the filtering process.

- an additional step has been added:

3b. Project onto the same affine subspace described in step 3 all the neighbors of $y_{i}$ at distance smaller than $\delta$. These points are no longer processed in the current iteration. $\delta$ must be smaller than $T$ so the affine subspace found in step 3 is a good approximation of the subspace associated to these points. The addition of step $3 \mathrm{~b}$ implies a maximum reduction of the computation time of the order of $(2 \delta+1)^{3}$. After experimental testing on several images (see Section 4 ) we have fixed $\delta=T / 2$.

- the number of iterations is controlled by $\varepsilon$. We fix it to 0.5 , which implies an average of 3 iterations for LLP2. The value was chosen after experimental testing on several images (see Section 4). 
Statistical analysis of the computation time of the algorithm with parameters fixed to $T=10$, $\delta=5$ and $\varepsilon=0.5$ ) has been performed using 100 images of the Berkeley test dataset [5]. The average computation time for these images has been computed for decreasing scale factors. The results, summarized in Table 2, show that the computation time has a non-linear dependency on the image size (at least for small scale factors).

$\begin{array}{cccc}\text { Scale } & \text { Size (pixels) } & \text { Size }_{\text {Size }} \text { (scale 1) } & \text { Time/Time }_{(\text {scale 1) }} \\ 1 & 154401 & 1 & 1 \\ 0.75 & 86400 & 0.56 & 0.59 \\ 0.5 & 38400 & 0.25 & 0.33\end{array}$

Table 2: 100 images from the Berkeley test dataset [5] have been zoomed down with decreasing scale factors. The size of the images is shown in the second column. The ratio between the size of the zoomed images and their original size is displayed in the third column. The last column shows the ratio between the average computation times of LLP2 for the zoomed images with respect to the computation time for the original images.

\section{Parameters Setting}

\subsection{Tests with $\mathrm{T}$}

The distance $T$ is used to select the neighboring points which take part in the PCA computation. The value of this parameter is not crucial, as illustrated in Figure 3 and Table 3. It must be remarked that $T$ is a distance in RGB space (not a distance between image pixels) and that in all our experiments 3 bytes per pixel are used to store color information, therefore the maximum Euclidean distance between two points in the color cube is $255 \times 3^{1 / 2}=441.67$.
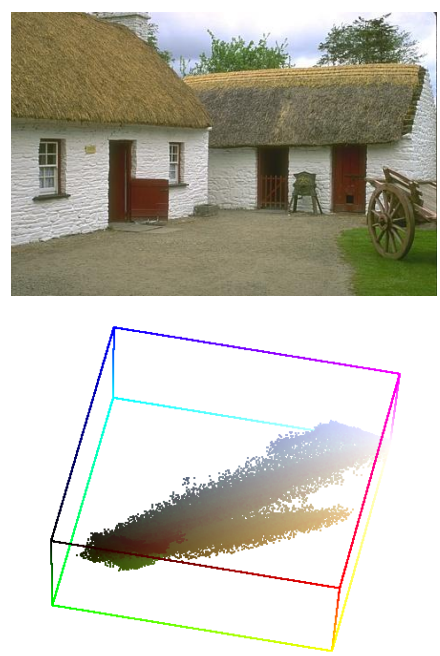
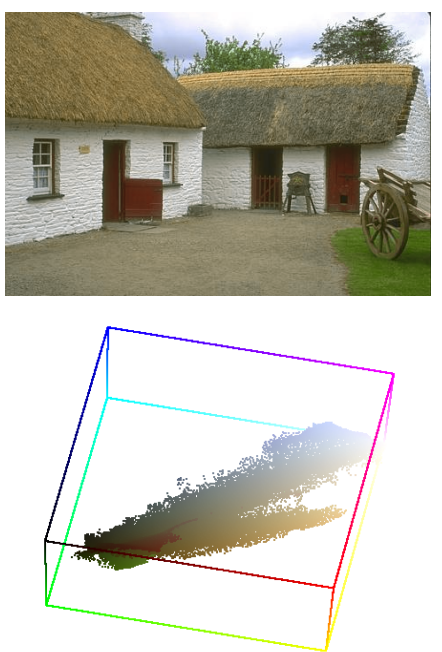
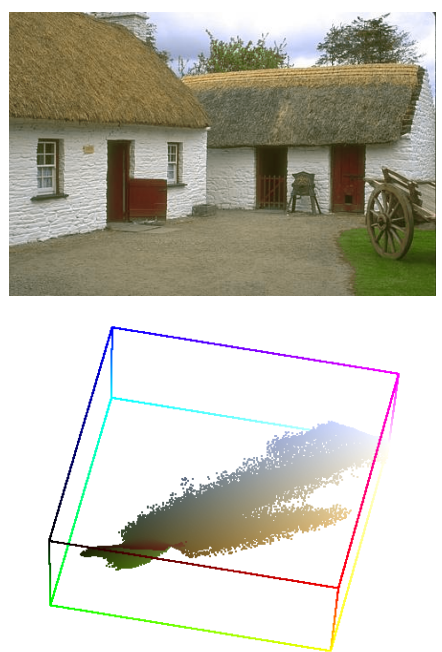
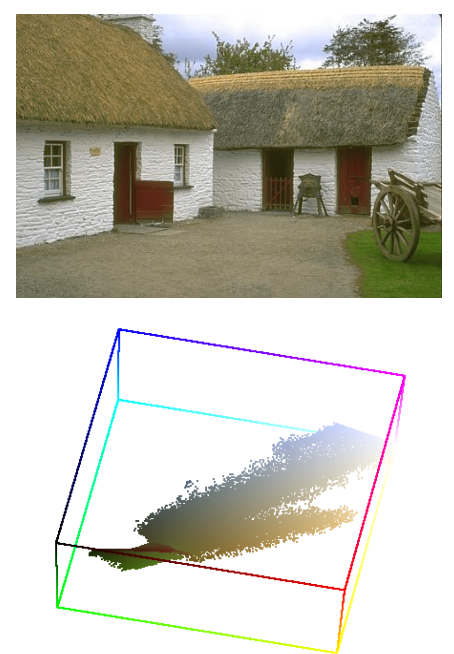

Figure 3: The original image (top-left) is filtered with LLP2, the radius $T$ of the filter being respectively 10, 15, 20. The corresponding clouds are in the bottom row. No color alteration is visible, which is consistent with the small variation of the cloud itself. This visual experiment is complemented by the quantitative analysis of Table 3.

In order to numerically verify the $2 \mathrm{D}$ filtering stability, the root mean square errors (RMSE) between the color clouds filtered with consecutive radii are shown in Table 3. The errors displayed in 
this table are the average errors over 100 images from the Berkeley test database [5]. The outcome of the experiment is decisive. The average distance between the cloud and its LLP2 filtered version remains small (less that 5), regardless of the value of the filtering radius $T$. In addition, the distances between filtered points for different values of the radius are also small (of the order of 2), proving that the filtered cloud is essentially the same regardless of the filtering parameter. This table therefore demonstrates the validity of the $2 \mathrm{D}$ manifold assumption. In all our experiments we have fixed parameter $T=10$.

$\begin{array}{ccc} & \text { RMSE } & \mathrm{dT}=5 \\ \mathrm{~T}=3 & 1.51 & 2.82 \\ \mathrm{~T}=5 & 2.43 & 2.54 \\ \mathrm{~T}=10 & 3.32 & 2.17 \\ \mathrm{~T}=15 & 3.80 & 2.03 \\ \mathrm{~T}=20 & 4.18 & 1.94 \\ \mathrm{~T}=25 & 4.48 & 1.76 \\ \mathrm{~T}=30 & 4.72 & 1.64\end{array}$

Table 3: Statistical results applying LLP2 $(\varepsilon=0.5, \delta=T / 2)$ on 100 images from the Berkeley test database [5]. First column: the radius $T$ for LLP. Second column: average RMSE between filtered point and original after applying LLP2. Third column: average RMSE between the final position of a point filtered by LLP2 with radius $T$, and the same point filtered by LLP2 with radius $T+d T$, where $d T=5$.

About the computation of RMSE. The root mean square errors (RMSE) displayed in Table 1 have been computed between two color clouds, not between two images:

$$
R M S E=\sqrt{\frac{1}{\operatorname{card}\left(\kappa_{0}\right)} \sum_{c \in \kappa_{0}} d^{2}(c, T(c))},
$$

where $\kappa_{0}$ is the set of different colors in the first input image and $T(c)$ is the color in the second image that corresponds to color $c$ in the first image (they belong to the same pixel in both images). $d(c, T(c))$ is the Euclidean distance in RGB space from color $c$ to color $T(c)$. Observe that the number of pixels associated to each color (its histogram value) is not taken into account.

Alternatively, the average distance between corresponding color points could have been computed:

$$
d_{\text {mean }}=\frac{1}{\operatorname{card}\left(\kappa_{0}\right)} \sum_{c \in \kappa_{0}} d(c, T(c)) .
$$

\subsection{Tests with $\delta$}

The parameter $\delta$ is used in the algorithm to speed up computations. Table 4 shows the average reductions in computation time for different values of $\delta$ (with fixed radius $T=10$ ), computed from 100 images of the Berkeley database [5]. The average RMSE between original and filtered images is also shown.

The results show that the computation time is reduced as $\delta$ increases, while the average RMSE remains small even for large values of the parameter. This result is explained by the smooth variations in orientation of the local regression planes computed for each RGB point, which makes it possible to approximate the local plane at a given point by planes computed at relatively far apart points. It must be remarked however that RMSE is an average measure over the set of points in a color 
cloud. Close observation of the clouds shows that when large values of $\delta$ are used the filtered color clouds tend to be composed of unconnected 2D manifolds, instead of forming a smooth surface (see Figure 4). For this reason we have chosen a compromise value $\delta=T / 2=5$ (since $T=10$ ) in all our experiments.

$\begin{array}{ccc} & \text { Time } / \text { Time }_{(\delta=0)} & \text { RMSE } \\ \delta=0 & 1 & 3.30 \\ \delta=1 & 0.25 & 3.30 \\ \delta=2 & 0.11 & 3.30 \\ \delta=3 & 0.065 & 3.31 \\ \delta=5 & 0.036 & 3.32 \\ \delta=7 & 0.03 & 3.37 \\ \delta=10 & 0.028 & 3.56\end{array}$

Table 4: The first column shows the ratio between the average computation time of the LLP2 algorithm $(T=10, \varepsilon=0.5)$ for different values of the speed-up parameter $\delta$ with respect to the computation time without acceleration $(\delta=0)$. The average RMSE with respect to the original images is also displayed. Averages were taken over 100 images in the Berkeley test dataset [5].

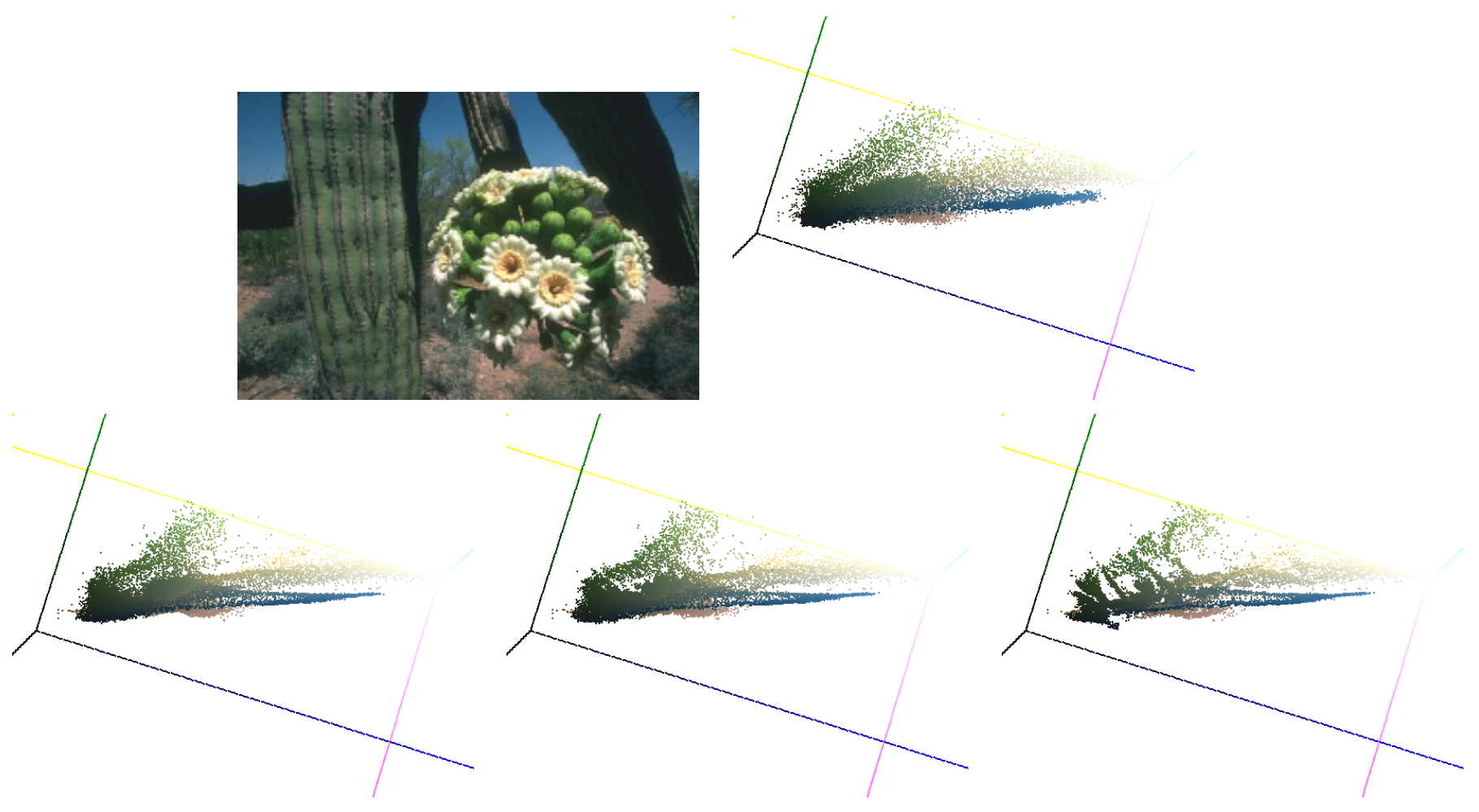

Figure 4: Top, original image and its color cube. The image is filtered with the LLP2 algorithm with $T=10, \varepsilon=0.5$ and increasing values of the speed-up parameter $\delta$. Bottom, from left to right, resulting cubes with $\delta=0$ (no acceleration), $\delta=5$ and $\delta=10$. For large values of $\delta$ (bottom-right) the filtered color clouds tend to be composed of unconnected 2D manifolds, instead of forming a smooth surface (bottom, left and center). 


\subsection{Tests with $\varepsilon$}

The parameter $\varepsilon$ is used to control the number of iterations of the algorithm. Table 5 displays the average number of iterations for 100 images in the Berkeley test database [5] for different values of $\varepsilon$. The average RMSE between original and filtered images is also shown. As the number of iterations (and therefore the computation time) increases the dominant 2D structures become more visible, at the expense of losing details of the color distribution (see Figure 5). As a consequence the difference (RMSE) with the original values increases. A compromise solution has been to set $\varepsilon=0.5$.

$\begin{array}{ccc} & \text { Number of iterations } & \text { RMSE } \\ \varepsilon=0.05 & 29.3 & 4.71 \\ \varepsilon=0.1 & 11.92 & 4.09 \\ \varepsilon=0.25 & 4.51 & 3.55 \\ \varepsilon=0.5 & 2.91 & 3.32 \\ \varepsilon=0.75 & 2.48 & 3.24 \\ \varepsilon=1 & 2.23 & 3.17\end{array}$

Table 5: The second column shows the average number of iterations of the LLP2 algorithm $(T=10$, $\delta=5)$ computed from 100 images in the Berkeley test dataset [5]. The average RMSE with respect to the original images is also displayed. As the number of iterations increases the difference with the original values increases.

\section{$5 \quad$ Preprocessing and Visualization of Results}

In the Overview section it was remarked that isolated colors in the RGB cube make an opaque obstacle to the perspective view of the cloud of RGB points. LLP2 recreates a 2D manifold from the unstructured cloud of points allowing for a better visualization of the colors. However, isolated colors are not eliminated by LLP2 (their number of neighboring colors is below threshold $K$ and therefore their original value is preserved) and they still hinder visualization of the processed cube.

In order to improve the visualization of the original and filtered RGB cubes the following procedure is applied:

1. Eliminate "isolated" colors from the original image. One color is labeled as "isolated" if its number of neighbors (number of color points at L1-distance $\leq r$ ) is below some threshold $n_{\min }$. Values $r=5$ and $n_{\min }=10$ are used in all our experiments.

2. Apply LLP2 on the remaining colors.

This procedure is used in the online demo and in the examples below. Results are displayed as follows:

- Original image.

- RGB cube of the original image after removal of isolated colors.

- RGB cube after processing with LLP2 the cube from the previous step.

- Filtered image, where pixels with "isolated" colors in the original image keep their original color. 


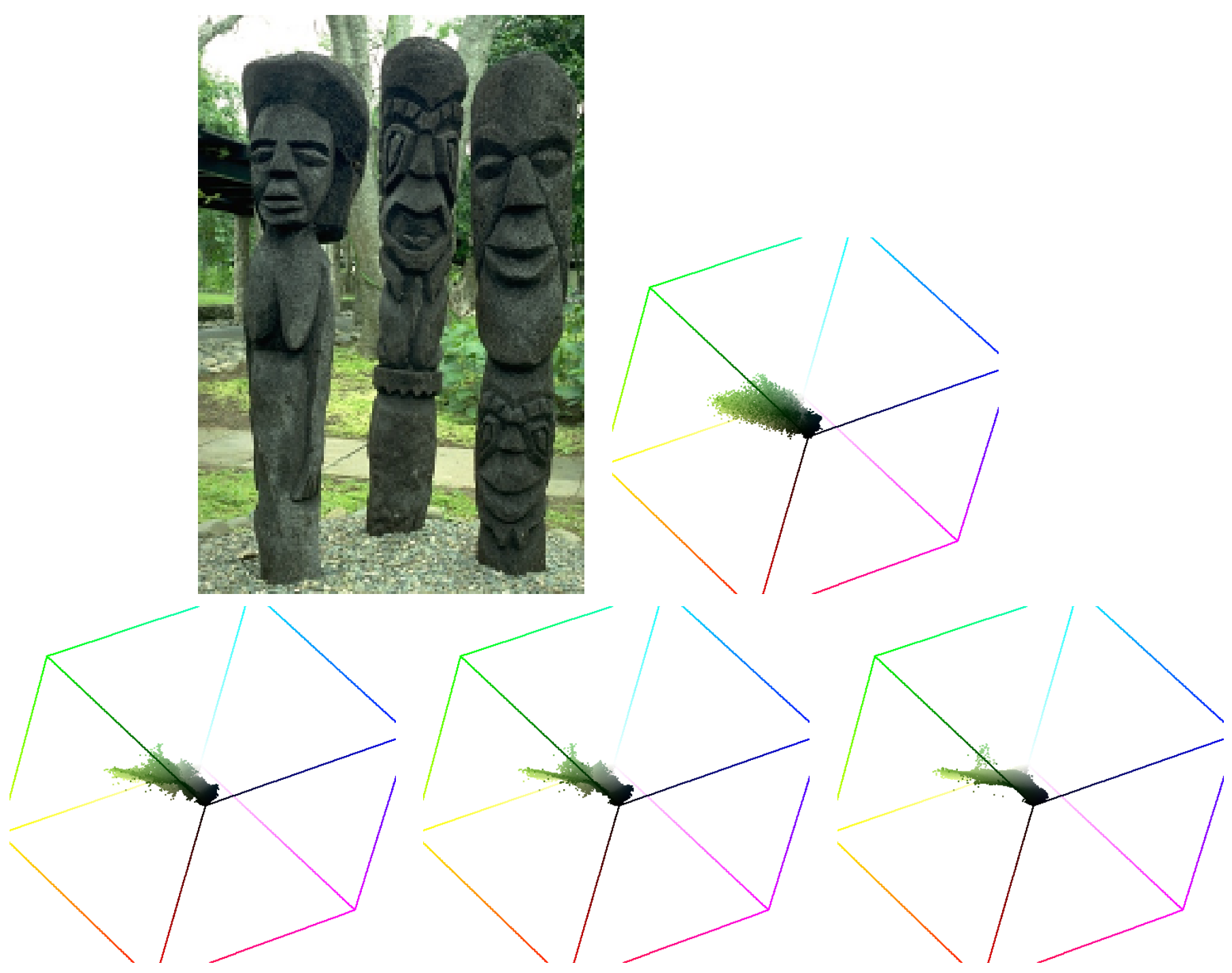

Figure 5: Top, original image and its color cube. The image is filtered with LLP2 with $T=10$, $\delta=5$ and increasing values of $\varepsilon$. Bottom, from left to right, resulting cubes with $\varepsilon=1, \varepsilon=0.5$ and $\varepsilon=0.05$. As the number of iterations increases the dominant $2 \mathrm{D}$ structures become more visible, at the expense of losing details of the color distribution.

Isolated color points are mainly located on boundaries, and they represent a small fraction of the total number of image colors. Tests with the 100 images of the Berkeley test dataset [5] show an average value of $2 \%$ isolated colors per image. An average of $1 \%$ image pixels correspond to isolated colors.

Figure 6 displays an original image, the resulting image after removal of isolated colors (pixels set to black), the filtered image and their corresponding color cubes.

\section{$6 \quad$ More Examples}

This section displays (see Figure 7) some results of the application of LLP2 (including removal of isolated points) on different images from Berkeley test dataset [5]. 

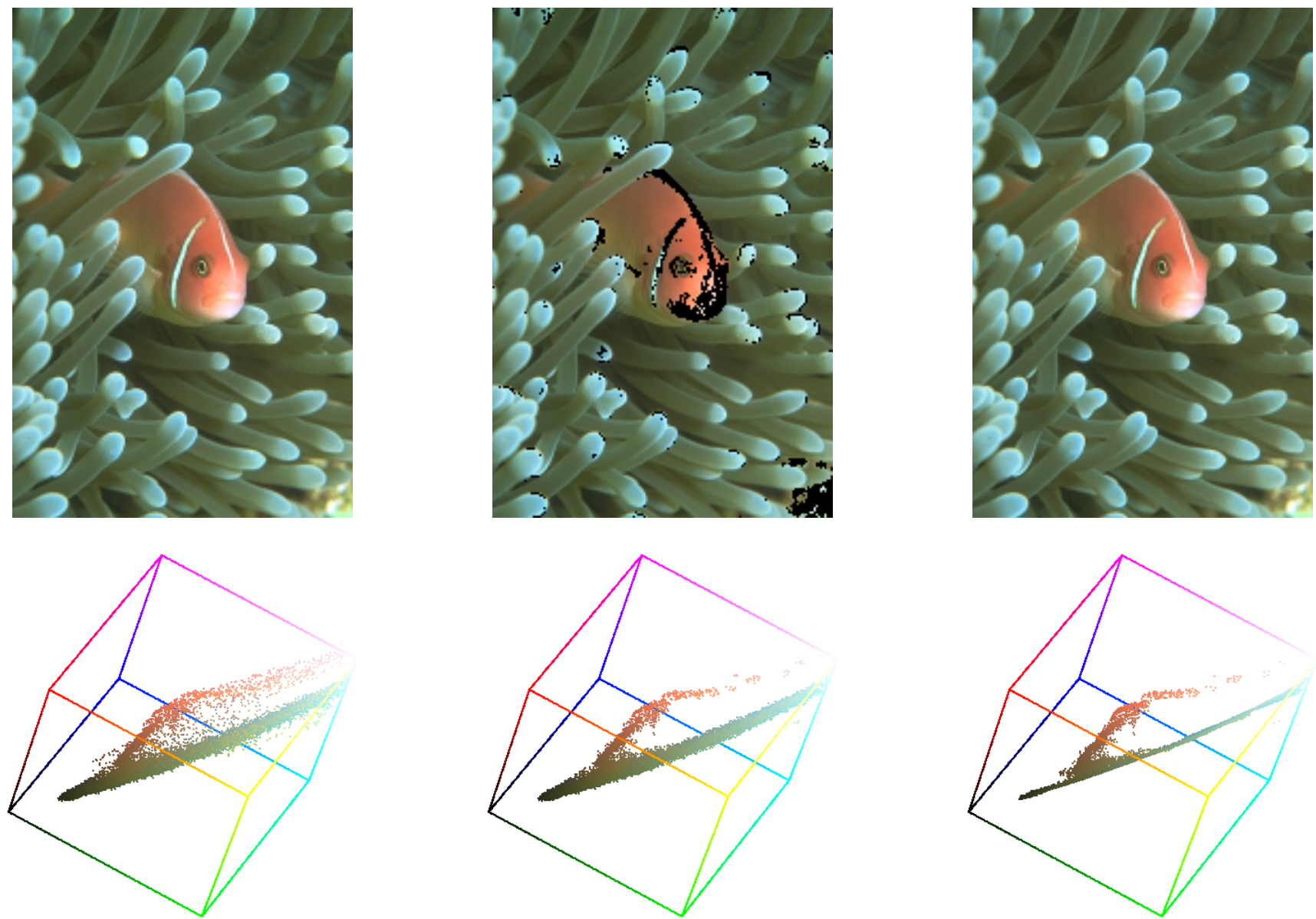

Figure 6: Top row. From left to right, original image, the same image after removal of isolated colors (associated pixels set to black) and filtered image. $6.02 \%$ of the colors have been removed $(3.84 \%$ of pixels). (This percentage is exceptionally high, the average removal rate being 1\%.) Bottom, from left to right, original color cube, cube after removal of isolated colors and result of LLP2 on this last cube.

\section{Color Density Visualization}

The 2D structure of color histograms permits a simple and reliable visualization tool (LLP2) recreating a 2D color manifold from the unstructured color cloud point. On this manifold the color density itself can be displayed by a brightness value proportional to the density of the color point.

Figure 8 shows another perspective of the filtered point clouds of the images in Figure 7, and also displays their histogram density (lighter grey levels indicate higher densities). The density is displayed in logarithmic scale, because a few color points can have a huge occupancy (the typical "blue sky effect") and therefore a linear scale would squeeze the density values. The density is only now visible because the filtered clouds are flat and the dense color points in direct sight.

\subsection{How the Color Density is Computed}

The density of a color point in RGB space is a measure of the occupancy of the space in a neighborhood of the point. Density values are computed as follows

$$
\rho(c)=\sum_{c_{s} \in \kappa} e^{-d^{2}\left(c, c_{s}\right) / h^{2}}
$$



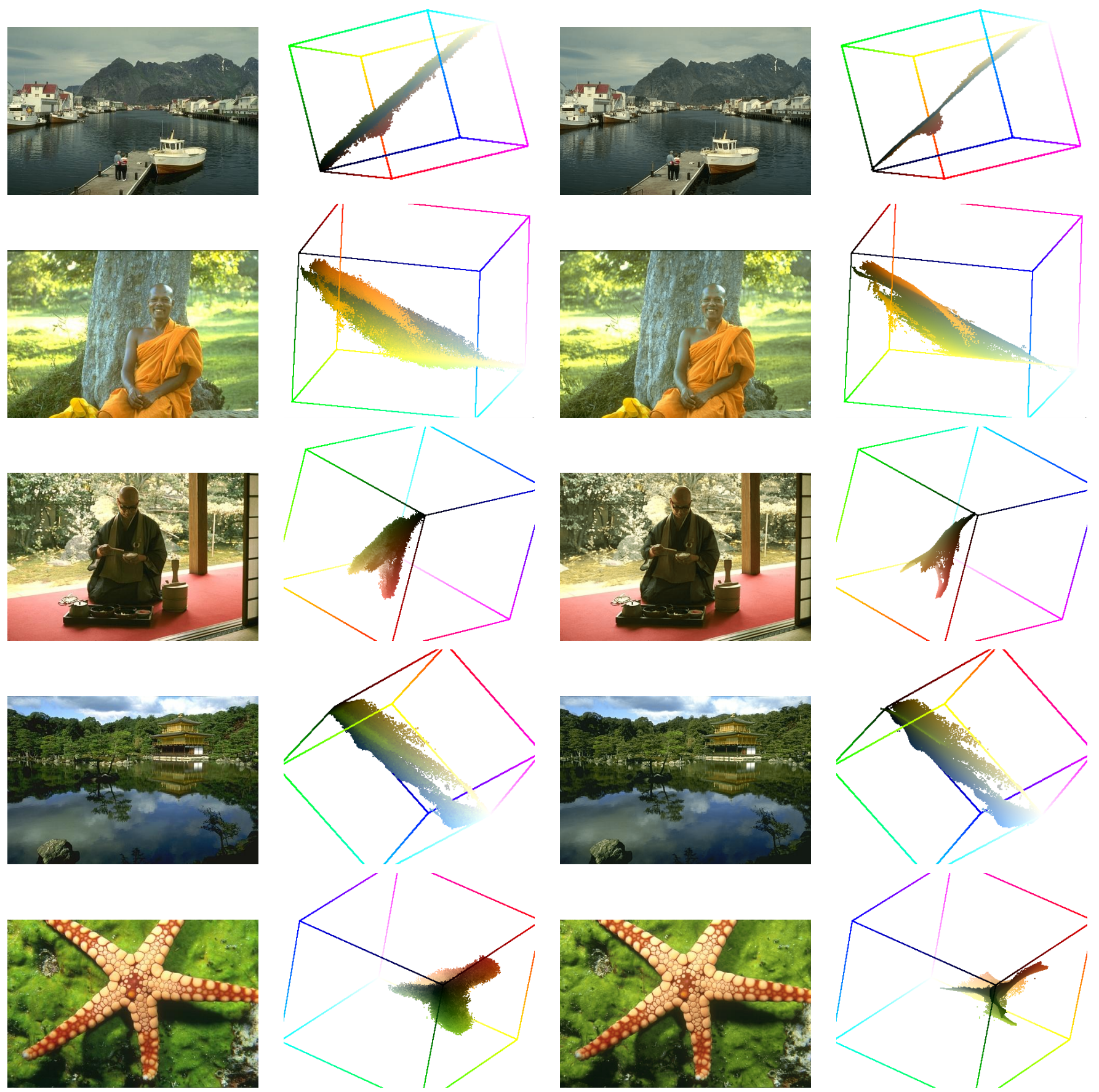

Figure 7: Five images, their original cloud, their filtered cloud by LLP2 $(T=10, \delta=5, \epsilon=0.5)$, and the image with the filtered colors. The filtered cloud is a 2D numerical manifold, being a steady state for LLP2. There is no conspicuous alteration in the color cloud when passing to 2D, and the image with filtered colors looks identical to the original.

where $c$ is a color in RGB space, $\kappa$ is the set of its neighbors at distance smaller or equal to $T, h$ is a weighting factor, and $d\left(c, c_{s}\right)$ is the Euclidean distance between colors $c$ and $c_{s}$ in RGB space. In this case each color is counted as many times as it appears in the image (number of pixels having the color value). $T$ and $h$ have been set to $T=5$ and $h=T / \sqrt{2}$ in all our experiments. 


\section{Image Credits}

All the images are from the Berkeley Segmentation Dataset and Benchmark².

\section{References}

[1] T. Buades, J.L. Lisani and J.M. Morel. The dimensionality of color space in natural images, Journal of the Optical Society of America A, 28(2), pp.203-209, 2011. http://dx.doi.org/10. 1364/JOSAA . 28.000203.

[2] D. Levin. The approximation power of moving least-squares, Mathematics of Computation, 67(224), pp.1517-1531, 1998. http://dx.doi.org/10.1090/S0025-5718-98-00974-0.

[3] J. Digne, J.M. Morel, C. Mehdi-Souzani and C. Lartigue. Scale Space Meshing of Raw Data Point Sets, Computer Graphics Forum, 2011. http://dx.doi.org/10.1111/j.1467-8659. 2011.01848.x.

[4] X. Huo and J. Chen. Local linear projection (LLP) Proceedings of First Workshop on Genomic Signal Processing and Statistics (GENSIPS), 2002.

[5] D. Martin, C. Fowlkes, D. Tal and J. Malik. A Database of Human Segmented Natural Images and its Application to Evaluating Segmentation Algorithms and Measuring Ecological Statistics, Proceedings of 8th International Conference on Computer Vision, pp.416-423, 2001. http://dx . doi.org/10.1109/ICCV.2001.937655.

\footnotetext{
${ }^{2}$ https://www.eecs.berkeley.edu/Research/Projects/CS/vision/bsds/
} 

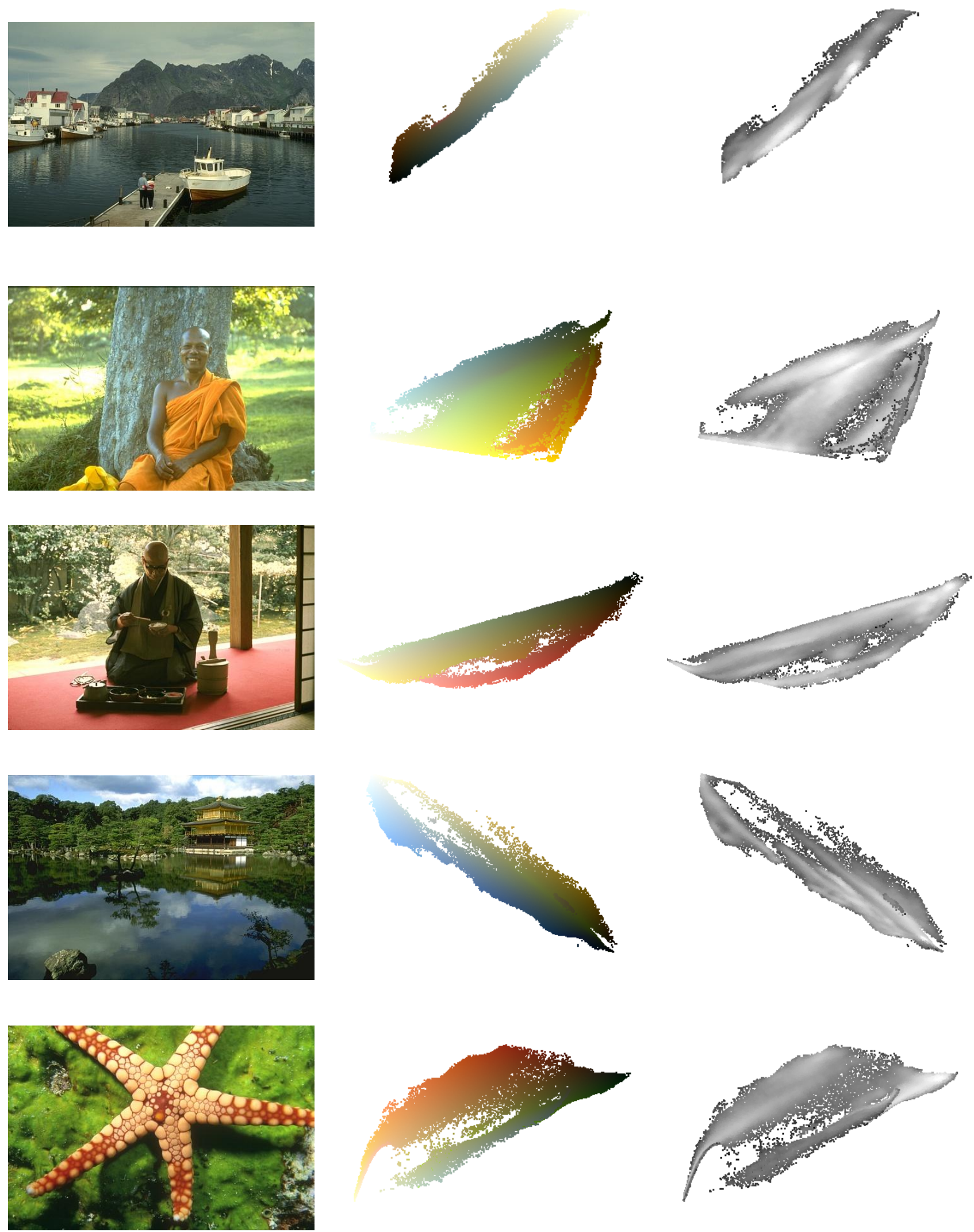

Figure 8: Densities of the filtered RGB cubes in Figure 7. Left: original image. Center: a view of the filtered RGB cube. Right: Same view for the density. The real density is now visible because the cloud has been flattened into a 2D manifold. Otherwise the dense color points would be hidden, being occluded by less dense ones. 